\title{
ELECTRICAL CONDUCTION BY PERCOLATION IN THICK FILM RESISTORS
}

\author{
F. FORLANI \\ Magneti Marelli, Divisione Electtronica FIVRE, 27100 PAVIA, Italy \\ and \\ M. PRUDENZIATI \\ Istituto di Fisica, Universitá di Modena, 41100 MODENA, Italy
}

(Received May 2, 1976)

\begin{abstract}
Thick film resistors are widely used in microelectronic devices, however the mechanism of electrical conduction in these resistors has not yet been fully understood. In particular the anomalous behaviour of the temperature coefficient of resistance (T.C.R.) vs. temperature for a purely ohmic resistor has not been explained. The anomaly is that the T.C.R. is negative at low temperatures, is zero around room temperature and becomes positive at higher temperatures.

This paper demonstrates that the electrical conduction mechanism in thick film resistors can be described by the electron percolation theory already proposed to explain charge transport in amorphous semiconductors. The thick film structure consists of conductive grains with a diameter of $0.1 \mu \mathrm{m}$ to $0.3 \mu \mathrm{m}$ separated by dielectric layers. Some of the conductive grains make contact through dielectric layers so thin that electrons are able to tunnel through the layers. The critical percolation path is through these grains. Experimental evidence is given which confirms that the resistance vs. temperature characteristics satisfactorily fit the conduction equation provided by the percolation theory.

The T.C.R. anomaly can be explained in the framework of this theory. The decay length of the electron wavefunction is shown to be lower than $370 \AA$ for a density of conductive grains in the film in the order of $10^{15} \mathrm{~cm}^{-3}$. Such a value is consistent with electron tunnelling through layers about $100 \AA$ thick.
\end{abstract}

\section{INTRODUCTION}

The use of thick film technology ${ }^{1}$ to fabricate resistors in hybrid circuits is well established. Nevertheless until now the physical models proposed to describe the electrical conduction mechanism in thick film resistors ${ }^{2-9}$ are unconvincing.

It is required to explain how a purely ohmic resistor can exhibit an anomalous behaviour of the temperature coefficient of the resistance (T.C.R.) vs. temperature: as shown in Figure 1, where the normalized conductivity is plotted vs. temperature, the T.C.R. of a thick film resistor is negative at low temperatures, it becomes zero around room temperature and turns positive at higher temperatures.

The first attempts to explain this anomaly 2,3 were based on the idea that conclusions about the conduction mechanisms in transition oxides could be extended to thick film resistors. With this approach the T.C.R. anomaly found a qualitative interpretation. However the model failed to provide a quantitative fitting to the experimental data on $\mathrm{PdO} / \mathrm{Ag}$ based resistors. A satisfactory fitting of those experimental data was obtained by $\mathrm{Kahan}^{4}$ whose approach consisted in completing the model elaborated by Brady ${ }^{5}$ and based on the conductivity characteristics of semiconductive oxide grains. Brady assumed the oxide grains to be submitted to a pressure applied by the glassy matrix and dependent upon the temperature. Kahan also took into account the contribution to the current flow due to metallic grains. One of us ${ }^{6}$ has already pointed out that Brady-Kahan model was based on six independent assumptions with no possibility for these to be checked independently. Moreover it was shown that Eqs. 1 and 2 for conductivity fitted the experimental data used by Kahan very well:

$$
\sigma \propto \exp -\left(\frac{T_{0}}{T}\right)^{1 / 4}
$$

at low temperatures, and

$$
\sigma \propto T^{-n} \exp -\frac{E}{k T}
$$






FIGURE 1 Normalized conductivity of a typical thick-film resistor vs. temperature.

at higher temperatures. In Eqs. (1) and (2), $T_{0}$ is a constant with dimensions of temperature and $n$ is a pure number of order $1 / 3$.

It was also noted ${ }^{7}$ that Eqs. (1) and (2) provide a good fit for experimental data concerning $\mathrm{Ir}-\mathrm{RuO}_{2}$ based resistors.

$\mathrm{Hill}^{8}$ has confirmed the validity of Eq. (1) to fit, at low temperatures, data on $\mathrm{Bi}_{2} \mathrm{Ru}_{2} \mathrm{O}_{7}$ based resistors and concluded that electrical conduction in thick film resistors can be attributed to hopping of charge carriers between states localized in a very narrow band. However, as already reported, ${ }^{9}$ the values of $T_{0}$ deducible from the experimental data are much lower than those expected by chargecarrier hopping mechanisms.

In the present paper we consider a proposal, already put forward, i.e. the electrical conduction in thick film resistors can be described by electron percolation, according to the theory initially proposed by Miller and Abrahams ${ }^{10}$ and used by Ambegaokar, Halperin and Langer ${ }^{11}$ to interpret conduction phenomena observed in disordered systems.

\section{ADAPTATION OF THE PERCOLATION MODEL FOR THICK FILM RESISTORS}

The percolation model assumes that the resistive material can be considered equivalent to a resistance network so that its over-all electrical conductivity can be written as

$$
\sigma=\frac{G_{c}}{\eta}
$$

where $G_{c}$ is some characteristic value of the conduc- 
tances in the network and $\eta$ is some characteristic length scale for the network. Let $G_{i j}$ be the conductance between any two directly interacting sites $i$ and $j$, where more resistors of the assumed network join together. Even though the explicit dependence of $G_{i j}$ on the material parameters varies according to the mechanism for the electron exchange between sites $i$ and $j$, it can be asserted that for a network, where the values of individual resistances vary over many orders of magnitude, $G_{c}$ in Eq. (3) represents the critical percolation conductance. In order to have a better understanding of the significance of $G_{c}$ consider the resistance network as composed of three parts: ${ }^{11}$

i) a set of isolated regions of high conductivity, each region consisting of a group of sites linked together by conductances with $G_{i j} \gg G_{c}$;

ii) a relatively small number of resistors with $G_{i j}$ of order $G_{c}$ connecting together a subset of high conductance regions as defined in (i);

iii) the remaining resistors with $G_{i j} \ll G_{c}$.

A thick film resistor can be represented by a resistance network with the characteristics described above. A thick film resistor is obtained ${ }^{1}$ through the firing of specially prepared screenable compositions at temperatures ranging from $750^{\circ} \mathrm{C}$ to $1150^{\circ} \mathrm{C}$. Such compositions are made of three main constituents: a conductive ingredient (generally, one or more conductive oxides as $\mathrm{PdO}, \mathrm{RuO}_{2}, \mathrm{NbO}_{5}$, etc.), a glassy content (generally a mixing of $\mathrm{PbO}, \mathrm{SiO}_{2}$, $\mathrm{B}_{2} \mathrm{O}_{3}, \mathrm{P}_{2} \mathrm{O}_{5}$, etc.) and an organic vehicle (butylcarbitol acetate, ethylcellulose, etc.). This last gives to the composition the thixotropy necessary for printing on to a ceramic substrate (generally, $96 \% \mathrm{Al}_{2} \mathrm{O}_{3}$ ) through a metal screen and is almost completely eliminated during the drying of the screened film before firing. The glassy content, on the contrary, remains in the film after firing and provides the adhesion of the film itself to the ceramic substrate. Even though after a correct firing the glass is mainly located at the interface between the film and the ceramic substrate, the as-fired film consists of a dense dispersion of small conductive grains (with diameters of $0.1 \mu \mathrm{m}$ to $0.3 \mu \mathrm{m}^{8}$ ) in a glassy matrix.

With this physical structure, a thick film resistor can be considered as consisting of: (1) a set of isolated regions with $G_{i j} \gg G_{c}$, corresponding both to simple conductive grains and to groups of conductive grains in purely ohmic contact; (2) a set of conductive grains in electrical contact through contact resistances having a non-zero value and consisting of resistors with $G_{i j}$ of the order of $G_{c}$. These form, together with at least a part of the first set, a critical subnetwork shorting out those resistors with $G_{i j} \ll G_{c}$ which correspond to higher contact resistances between conductive grains.

The model we assume for the conduction in thickfilms is, first of all, based on the following considerations: the conductances of order $G_{c}$ determine and dominate the resistance of the network electrically equivalent to the thick-film resistor. This means that whether the conductive grains exhibit metallic or semiconductive conduction mechanisms becomes unimportant. In order to elucidate $G_{c}$, it is assumed that conduction is due to electron hopping between the percolation sites because of tunnelling effect through the potential barriers provided by the glass into which the conductive grains are embedded.

Using, for $G_{c}$, the same expression given by Ambegaokar et al. ${ }^{11}$ for the conduction in amorphous materials, even though in this case the percolation sites are made of conductive grains instead of electron traps, we have

$$
G_{c}=\frac{e^{2}}{k T} \Gamma_{c}
$$

where $e$ is the electron charge and $\Gamma_{c}$ is the average transition rate from sites to sites along the critical percolation path. It is given by

$$
\Gamma_{c}=\gamma_{0} \exp -\left(\frac{4 v_{c} \alpha^{3}}{\rho_{0} k T}\right)^{1 / 4}
$$

where $\gamma_{0}$ is a constant depending upon the electron--phonon coupling strength, the phonon density of states and other elastic properties of the material, $\nu_{c}$ is a dimensionless constant, $\rho_{0}$ is the density of percolation sites per unit volume and unit energy and $\alpha^{-1}$ is a length whose physical meaning is associated with the tunnelling mechanism from one percolation site to the other, and therefore to the average diameters of the conductive grains along the percolation path. In other words, the meaning of $\alpha^{-1}$ is the basic difference between the conduction model by electron percolation applied to amorphous semiconductors and to thick films. While in amorphous semiconductors we must expect a value of $\alpha^{-1}$ of the order of a few $\AA$, in thick films its value must be comparable with the diameter of the conductive grains, i.e. some $100 \AA$. By assuming the validity of Eq. (5) in the case of thick film resistors, we consider the charge on a single grain concentrated at the centre of the grain and the separation between percolation sites a bit larger than the grain diameter. 
Thus, from Eqs. (3), (4) and (5), the conductivity of a thick-film resistor, is given by

$$
\sigma \propto \frac{\beta}{T} \exp -\left(\frac{T_{0}}{T}\right)^{1 / 4}
$$

where

$$
\begin{gathered}
\beta=\frac{e^{2} \gamma_{0}}{k} \\
T_{0}=\frac{4 \nu_{c} \alpha^{3}}{\rho_{0} k}
\end{gathered}
$$

and where we have implicity assumed that factor $\eta^{-1}$ in Eq. (3) is a relatively slowly varying function of the parameters of the network and that the dominant variation of $\sigma$ is contained in $G_{c}$.

It can be seen that the resistance, $R$, of a thickfilm resistor and associated T.C.R. is given by

$$
\begin{aligned}
& R \propto \frac{T}{\beta} \exp \left(\frac{T_{0}}{T}\right)^{1 / 4} \\
& \text { T.C.R. } \equiv \frac{1}{R} \frac{\mathrm{d} R}{\mathrm{~d} T}=\frac{1}{T}\left[1-\frac{1}{4}\left(\frac{T_{0}}{T}\right)^{1 / 4}\right]
\end{aligned}
$$

and T.C.R. becomes zero for a temperature such that

$$
T_{\min }=\frac{T_{0}}{256}
$$

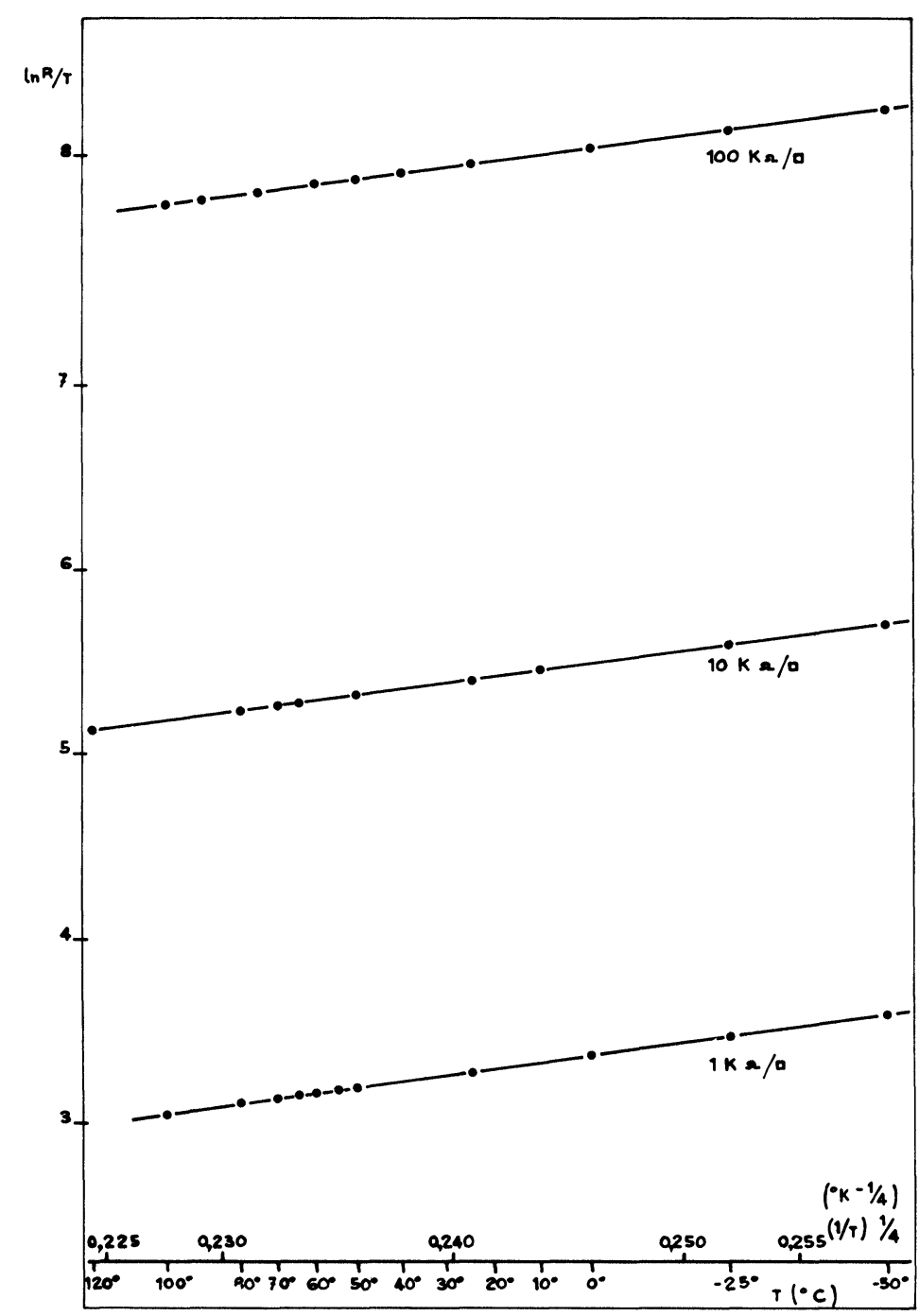

FIGURE 2 Matching between theory and experiments for thick-film resistors with the same aspect ratio and with different sheet resistivities. 
This temperature corresponds to the minimum value acquired by the normalized resistance, $R / R_{\min }$, vs. temperature as shown in Figure 3.

\section{EXPERIMENTAL EVIDENCES}

A set of measurements was performed on $\mathrm{Bi}_{2} \mathrm{Ru}_{2} \mathrm{O}_{7}$ based thick film resistors. The connections to these resistors were provided by screen and fired PtAubased conduction films.

All the resistors had a constant width of $2 \mathrm{~mm}$, they were printed at the same nominal thickness and fired under the same temperature profile.

TABLE I

Comparison to check the consistency of percolation theory applied to thick-films

\begin{tabular}{lll}
\hline $\begin{array}{l}\text { Sheet resistivity } \\
(\Omega / \mathrm{sq})\end{array}$ & $\begin{array}{l}T_{\mathrm{min}} \\
\left(=T_{\mathrm{o}} / 256\right)\end{array}$ & $\begin{array}{l}\left(T_{\text {min }}\right)_{\text {experimental }} \\
\left({ }^{\circ} \mathrm{C}\right)\end{array}$ \\
\hline 1,000 & 59.4 & 60 \\
10,000 & 67.9 & 70 \\
100,000 & 71.2 & 75 \\
\hline
\end{tabular}

In Figure $2 \ln R / T$ is plotted vs. $(1 / T)^{1 / 4}$ for different sheet resistivities. In Figure 3 the plot of $R / R_{\min }$ vs. $T$ is shown.

In Table I the values of $T_{\min }$ calculated using Eq. (11) from the values of $T_{0}$ obtained from the slopes of the straight lines in Figure 2 and the experimentally derived values of $T_{\min }$ are compared; the agreement is good.

\section{DISCUSSION}

The behaviour of T.C.R. with temperature expressed by Eq. (10) indicates a negative infinity in the temperature coefficient as the absolute temperature tends to zero and a null temperature coefficient for the absolute temperature going to infinity. This implies that the T.C.R. must exhibit a maximum for some temperature larger than $T_{0} / 256$. An evaluation of the derivative of Eq. (10) indicates that such a maximum is to be found at a temperature of $T_{0} /(3.2)^{4}$. In practice, it means that a resistor having a null temperature coefficient at room temperature $\left(300^{\circ} \mathrm{K}\right)$, and consequently a value of $T_{0}=76800^{\circ} \mathrm{K}$ (see Eq. (11)), should exhibit a maximum T.C.R. at $459^{\circ} \mathrm{C}$, i.e. at such a high

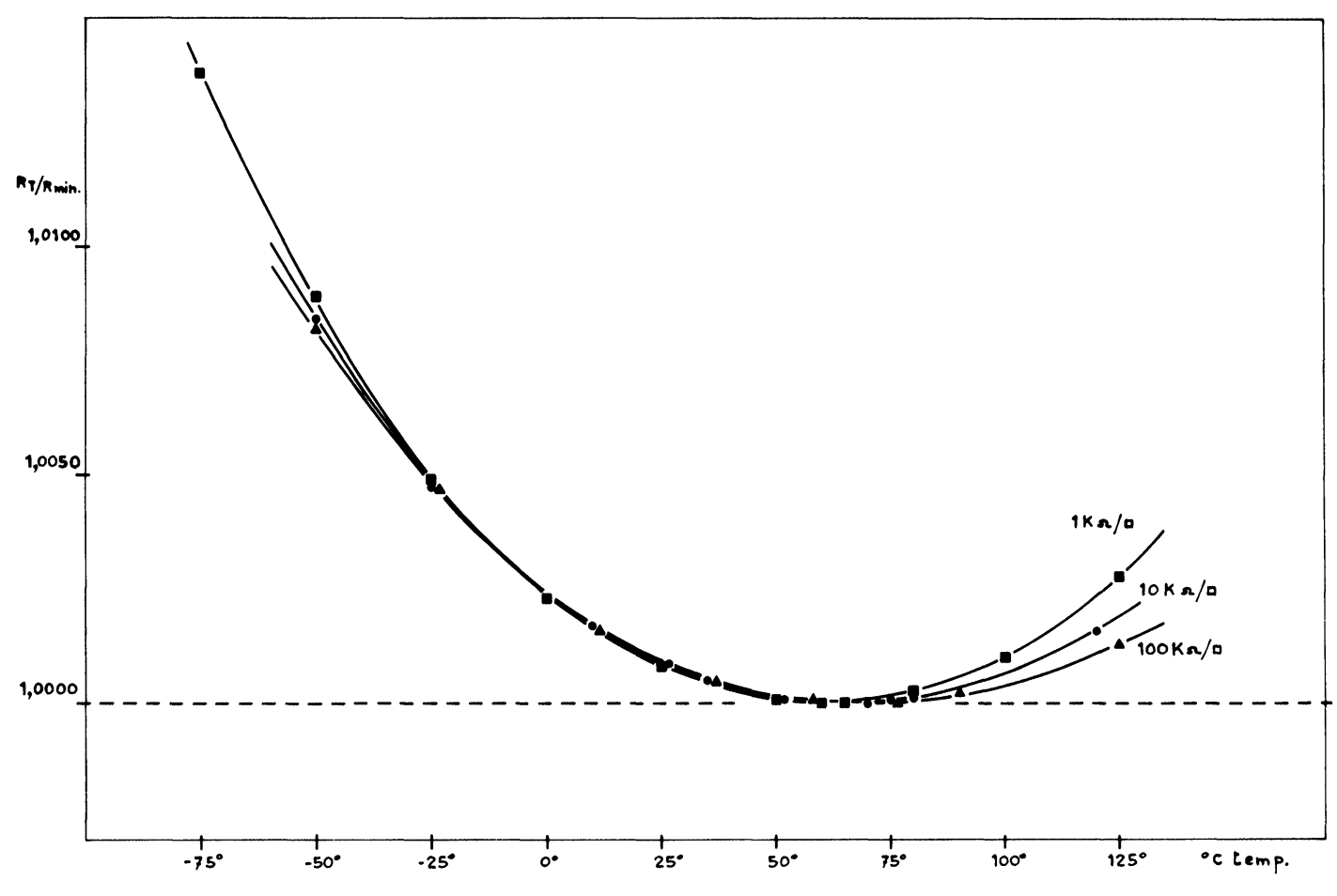

FIGURE 3 Plot of normalized resistance vs. temperature. 
temperature that it cannot be checked by experiments. The existence of a maximum in the T.C.R. at a temperature higher than $T_{0} / 256$ explains the lower gradient observed in the range of the high temperatures. On the other hand, the derivative indicates that for $T<T_{0} /(3.2)^{4}$ we have d(T.C.R.) $/ \mathrm{d} T>0$, as confirmed by experimental evidence. (8)

For a value of $T_{0}$ of $76800^{\circ} \mathrm{K}$ we obtain from Eq.

$$
\alpha^{3}=\frac{6.62(\mathrm{eV})}{4 \nu_{c}} \cdot \rho_{0}
$$

and for $\nu_{c} \simeq 4$

$$
\alpha^{3}=4.138 \times 10^{-1} \rho_{0}
$$

We can evaluate $\rho_{0}$ from the experimental measurements on the diameter of the conductive grains in the films reported by $\mathrm{Hill}^{8}$ for $\mathrm{Bi}_{2} \mathrm{Ru}_{2} \mathrm{O}_{7}$ based resistors. The reported diameters of $0.1 \mu \mathrm{m}$ to $0.3 \mu \mathrm{m}$ correspond to a grain density, $n$, of $10^{14} \mathrm{~cm}^{-3}$ to $10^{15} \mathrm{~cm}^{-3}$. As already pointed out, ${ }^{11}$ the grain density is related to the sites per unit volume and per unit energy, $\rho_{0}$, by

$$
n=2 \rho_{0} E_{\max }
$$

where $E_{\max }$ is the maximum energy distance from the Fermi energy, within which the electron density of states has to be constant. A more recent analysis performed by Pollak et al. ${ }^{12}$ has demonstrated that sites with an energy distance far away from the Fermi level contribute in a negligible way to the charge transport at the percolation path. In other words Eq. (14) must be written as follows

$$
n<2 \rho_{0} E_{\max }
$$

Then, the maximum energy distance from the Fermi level can be expressed by ${ }^{11}$

$$
E_{\max }=k T \ln \frac{\gamma_{0}}{\Gamma_{c}}
$$

and from Eq. (5)

$$
E_{\max }=k T^{3 / 4} T_{0}^{1 / 4}
$$

At room temperature for the resistor under consideration (with T.C.R. $=0$ at $300^{\circ} \mathrm{K}$ ) we obtain $E_{\max }=$ $0.103 \mathrm{eV}$. Then for a density of conductive grains of $10^{14} \mathrm{~cm}^{-3}$ to $10^{15} \mathrm{~cm}^{-3}$ :

$$
\rho_{0}>4.85 n \mathrm{~cm}^{-3} \mathrm{eV}^{-1}
$$

where $n$ is $10^{14} \mathrm{~cm}^{-3}$ to $10^{15} \mathrm{~cm}^{-3}$ and from Eqs.

(12) and (13)

$$
\alpha^{-1}<(370 \text { to } 790) \AA
$$

Such a length is quite consistent with the discussed meaning of $\alpha^{-1}$ and with a diameter of the conductive grains on the order of $1000 \AA$ to $3000 \AA$.

Finally, it should be noted that the three straight lines of Figure 2 are almost parallel. The values of $T_{0}$ are only weakly dependent upon the sheet resistivity of the film (see Table I). The approach discussed here and based on a conduction mechanism by electron percolation does not allow us to determine the proportionality factor in Eq. (9).

\section{CONCLUSIONS}

The electron percolation theory as proposed by Ambegaokar et al.$^{11}$ provides a very simple and credible interpretation of the conduction mechanism in a thick-film resistor. The apparently anomalous T.C.R. vs. temperature behaviour finds an immediate explanation in the frame of this theory. It has been checked that the matching between theory and experiments holds also for resistor compositions other than $\mathrm{Bi}_{2} \mathrm{Ru}_{2} \mathrm{O}_{7}$ based ones as the extended series of tests was limited to $\mathrm{Bi}_{2} \mathrm{Ru}_{2} \mathrm{O}_{7}$ based resistors due to their stability and reproducibility.

It is not surprising that a theory dealing with disordered systems can be applied to thick-film structures. Moreover, further investigations, still underway, indicate that the assumption of a site density, $\rho_{0}$, constant through the film and expressing an average order in the disordered configuration, is only a first order approximation.

At least in the range of high sheet resistivity, the T.C.R.'s are dependent upon resistor aspect ratios and such a dependence might find an explanation in the frame of electron percolation theory by introducing some unhomogeneity in the site density. However, more work is necessary to confirm these preliminary observations.

\section{ACKNOWLEDGEMENT}

The authors wish to thank A. Cattaneo for the preparation of the films and for the measurements of the conduction characteristics.

\section{REFERENCES}

1. C. A. Harper, Handbook of Thick-Film Hybrid Microelectronics, McGraw-Hill, N.Y. (1974).

2. E. H. Melan and A. H. Mones, "The glaze resistor: its structure and reliability", Proc. IEEE Electron. Compon. Confer., Washington D.C. (1964). 
3. E. H. Melan, "Stability of Palladium Oxide resistive glaze films", Microelectronics \& Reliability, 6, 53 (1967).

4. G. J. Kahan, "Equivalent circuit for conductivitytemperature characteristics of $\mathrm{PdO} / \mathrm{Ag}-\mathrm{Pd}$ glaze resistors", IBMJ. Res. \& Dev., 15, 313 (1971).

5. L. J. Brady, "Mechanism of conduction in thick-film cermet resistors", Proc. IEEE Electron. Compon. Confer. Washington D.C. (1967).

6. F. Forlani, "Meccanismi di conduzione in film resistivi depositati per serigrafia", Alta Frequenza, 41, 921 (1972).

7. F. Forlani and M. Prudenziati, "Meccanismi di conduzione in film resistivi depositati per serigrafia", Comm. at 59th National Confer. Italian Phys. Soc., Florence (1973).
8. R. M. Hill, "Conduction theory applied to thick film resistors", IERE Conf. Proc., 31, 251 (1975).

9. F. Forlani, "Relative merits of thin film and thick-film technology", Comm. at 3rd Intern. Confer. on Thin Films, Budapest (1975); Thin Solid Films, (to be published).

10. A. Miller and E. Abrahams, "Impurity conduction at low concentrations", Phys. Rev., 120, 745 (1960).

11. V. Ambegaokar, B. I. Halperin and J. S. Langer, "Hopping conductivity in disordered systems", Phys. Rev., B4, 2612 (1971).

12. M. Pollak, M. L. Knotek, H. Kurtzman and H. Glick, "D.c. conductivity of amorphous germanium and the structure of the pseudogap", Phys. Rev. Letts., 30, 856 (1973). 

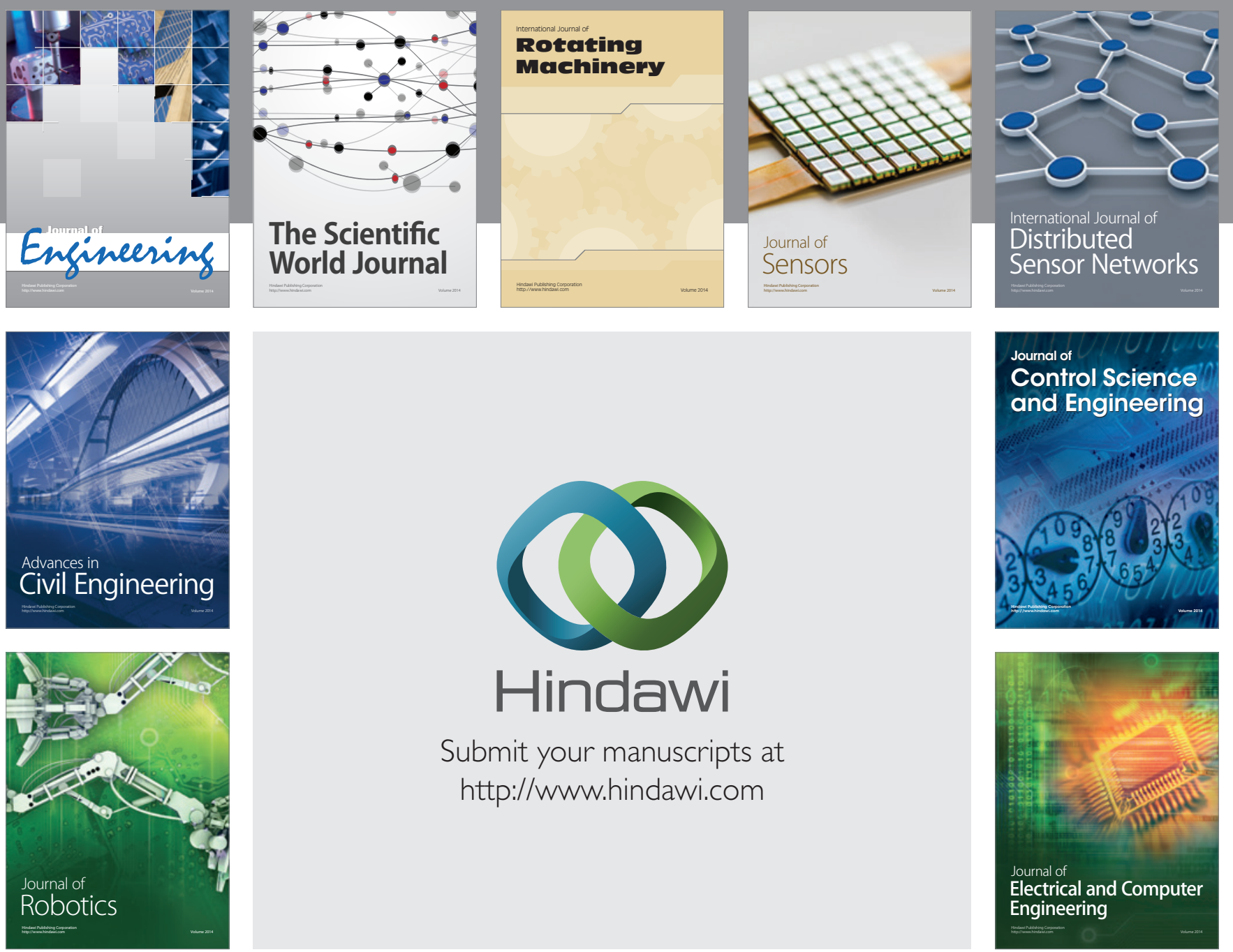

Submit your manuscripts at

http://www.hindawi.com
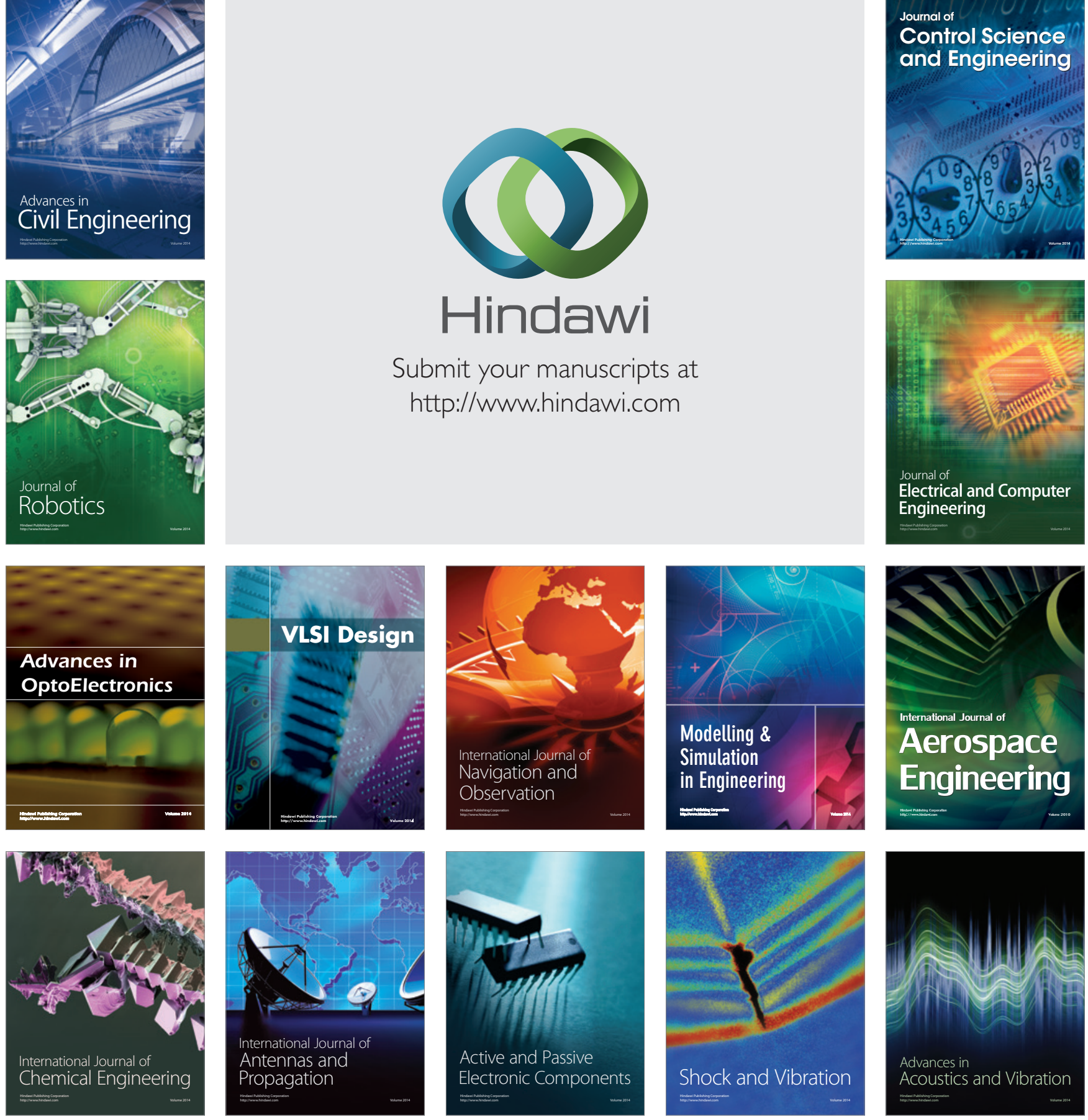